\title{
Range of Motion According to the Fusion Level after Lumbar Spine Fusion: A Retrospective Study
}

\author{
Se Jin $\mathrm{Choi}^{1}$, Ji Won Moon ${ }^{1}$, Dalsung Ryu ${ }^{1}$, Chang Hyun $\mathrm{Oh}^{2}$, Seung Hwan Yoon ${ }^{1}$ \\ ${ }^{1}$ Department of Neurosurgery, Inha University School of Medicine, Incheon, \\ ${ }^{2}$ Department of Neurosurgery, Guri Cham Teun Teun Hospital, Guri, Republic of Korea
}

Corresponding author: Seung Hwan Yoon Department of Neurosurgery, Inha University School of Medicine, 27, Inhang-ro, Jung-gu, Incheon 22332, Republic of Korea Tel: +82-32-890-2370 Fax: +82-32-890-2374 E-mail: nsyoon@gmail.com

Received: September 4, 2018 Revised: September 12, 2018 Accepted: September 12, 2018
Objective: The purpose of this study was to compare the range of motion (ROM) after spinal fusion according to the levels of lumbar spinal fusion (L4/5 and/or L5/S1). Methods: One hundred fifty-six patients were included in this study after undergoing thoracolumbar or lumbar fusion from April 2010 to December 2016. All patients had a numerical rating scale less than 4 . We categorized the patients according to the fusion level for statistical purposes: 86 patients in group I with L4/5 or L3/4/5 fusion, 24 in group II with only L5/S1 fusion, 34 in group III with L4/5/S1 or L3/4/5/S1 fusion, and 12 in group IV with T10-S1 fusion for lesions. The ROM was evaluated by physicians of the rehabilitation medicine department using a blinded method, and the result was compared between each group. The student t-test was used in the statistical analysis. Results: The ROM of lumbar flexion was not different between each group, except for groups I and IV $(p=0.038)$. The ROM of lumbar extension was statistically affected by fusion at the $L 4 / 5$ or $L 5 / S 1$ level. The ROM of lumbar lateral flexion had a tendency of being affected by fusion at the L4/5 or L5/S1 level. The ROM of lumbar lateral rotation was not affected by fusion at the $L 4 / 5$ or L5/S1 level. Conclusion: The results suggest that the lower lumbar segments ( $L 4 / 5$ and especially L5/S1) contribute to spinal ROM (extension and lateral flexion), but these segments alone do not play significant roles in spinal flexion movements.

Key Words: Lumbar vertebrae; Range of motion, articular; Spinal fusion

\section{INTRODUCTION}

The lower portion of the spine bears the most weight and allows for the greatest amount of motion ${ }^{4}$. Lumbar spine motion is very complex because of the need for both stability and mobility. The motion segment of the spine allows for motions within a restrained range and provides stability ${ }^{6}$. In the lower lumbar spine and lumbosacral region, flexion and extension are the primary motions. Lateral bending occurs mostly in the upper lumbar segments of L2/3, L3/4, and L4/5 rather than $\mathrm{L} 5 / \mathrm{S} 14)$. Biomechanical studies have reported on each range of motion (ROM) of the normal lumbar spine and disease lumbar spine, but few studies have investigated the ROM of patients after lumbar fusion ${ }^{1,4,20)}$. Physicians and patients recognize that fusion of the lumbar spine results in restricted range of all motions. Although this has been established in many animal or cadaveric studies, it has not been confirmed in humans ${ }^{1,3,8)}$. The main focus after fusion is on the partial ROM of the adjacent segment, and whole spine mobility has not attracted much interest until now. In the clinical setting, limitations of spinal $\mathrm{ROM}$ are a major problem directly related to the effects of activities on daily living and the disability rating involved with medical insurance. Herein, we compared the ROM after spinal fusion according to the levels of lumbar spinal fusion (L4/5 and/or $\mathrm{L} 5 / \mathrm{S} 1$ ).

\section{MATERIALS AND METHODS}

\section{Study Design and Population}

This retrospective study was conducted by reviewing the medical charts of all patients after thoracolumbar or lumbar fusion in a single university hospital from April 2010 to December 2016. All patients underwent spinal fusion by a single neurosurgeon. Among these patients, only those with more than 6 months of follow-up and those with an assessment of the ROM for the disability rating of medical insurance were included. The exclusion criteria of the study were patients who had a numerical rating scale (NRS-11) more than 4 and those with an Oswestry Disability Index (ODI) score of 20. The NRS-11 and ODI scores were determined by one nurse and one neurosurgeon. 
All patients were categorized into 4 groups according to the lumbar fusion levels as follows: group I, fusion at the $\mathrm{L} 4 / 5$ or L3/4/5 level ( $\mathrm{n}=86$ ); group II, fusion at the L5-S1 level $(\mathrm{n}=24)$; group III, fusion at the L4/5-S1 or L3/4/5-S1 level ( $\mathrm{n}=34)$; and group IV, fusion at other levels, such as T10/11/12-L1/2/3/ 4/5-S1, T12-L1/2/3/4/5, L1/2/3/4/5, L2/3/4/5-S1, and L3/4/5-S1 $(n=12)$. Because the normal ROM of the lumbar spine was difficult to determine in the clinical setting, we considered group IV (fusion at more than the L3 level) as the control group in order to compare it with patients who underwent fusion at the L4/5 or L5-S1 level.

\section{Follow-up}

All surgical treatment and postoperative care for 6 months were conducted in the department of neurosurgery, but the checkups for ROM of the lumbar spine were conducted in the department of rehabilitation. All lumbar spines were examined for the passive restricted point of ROM by specialists in rehabilitation after at least 6 months postoperatively. The specialists in rehabilitation had access to the patients' medical records, but the examination of ROM was performed in a blinded manner without reference to the patients' medical information, such as sex, age, fusion levels, and medical insurance issues. ROM of the lumbar spine was measured in degrees $\left({ }^{\circ}\right)$ in flexion, extension, lateral bending to the left and right sides, and lateral rotation to the left and right sides.

\section{Statistical Analysis}

Statistical differences between groups were determined by using the Student t-test, and SPSS software (IBM Corp., Armonk, NY, USA) was used to perform statistical analysis. Statistical significance was considered as $\mathrm{p}<0.05$.

\section{RESULTS}

One hundred fifty-six patients were included in this study (Table 1). ROMs of lumbar flexion were $29.48 \pm 12.63^{\circ}$ in group I, $32.08 \pm 13.01^{\circ}$ in group II, $31.43 \pm 12.90^{\circ}$ in group III, and $26.89 \pm 12.90^{\circ}$ in group IV (Fig. 1A). A statistical difference in the ROM of lumbar flexion was not observed between groups, except for groups I and IV ( $\mathrm{p}=0.038)$. ROMs of lumbar extension were $12.33 \pm 6.40^{\circ}$ in group I, $13.96 \pm 5.10^{\circ}$ in group II, 12.68 $\pm 5.52^{\circ}$ in group III, and $12.50 \pm 6.91^{\circ}$ in group IV (Fig. 1B). The ROM of lumbar extension was statistically affected by fusion at the L4-5 or L5-S1 level (all $\mathrm{p}<0.05$, except for in the comparison between group I and group II with a p-value of 0.387). Respective ROMs of lumbar lateral flexion in bending to the left and right sides were $18.37 \pm 6.66^{\circ}$ and $19.30 \pm 6.29^{\circ}$ in group I, $19.38 \pm 5.17^{\circ}$ and $20.00 \pm 4.89^{\circ}$ in group II, $19.11 \pm$ $4.72^{\circ}$ and $20.18 \pm 4.61^{\circ}$ in group III, and $17.22 \pm 7.12^{\circ}$ and 15.83

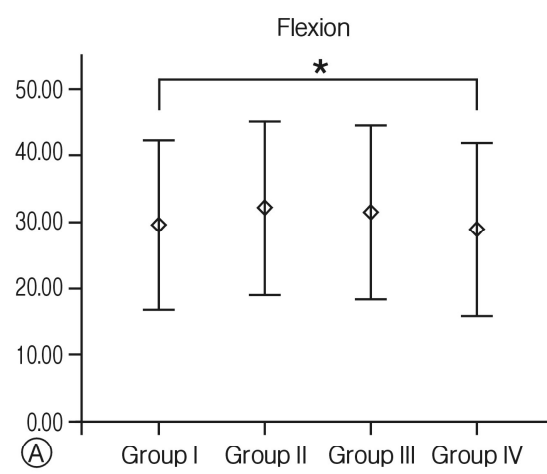

Lateral flexion on the left side

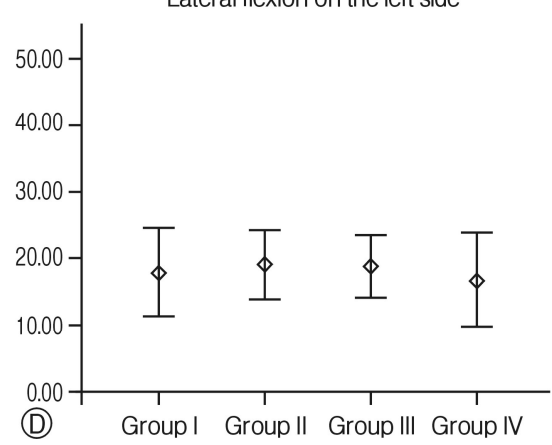

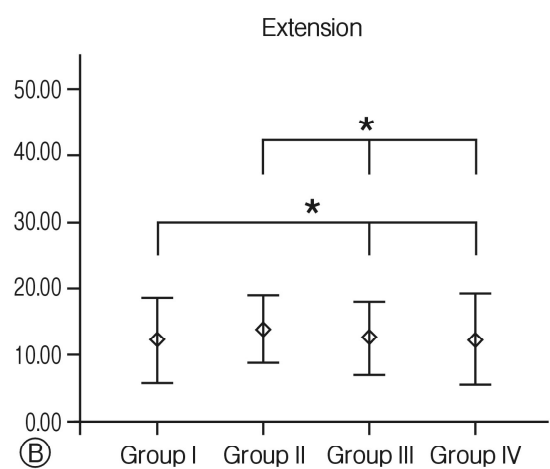

Rotation on the right side

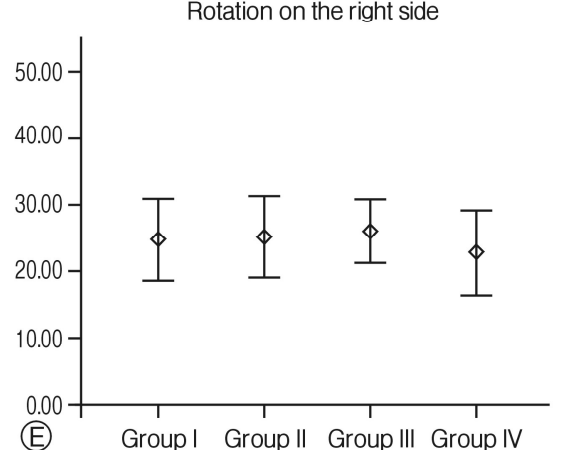

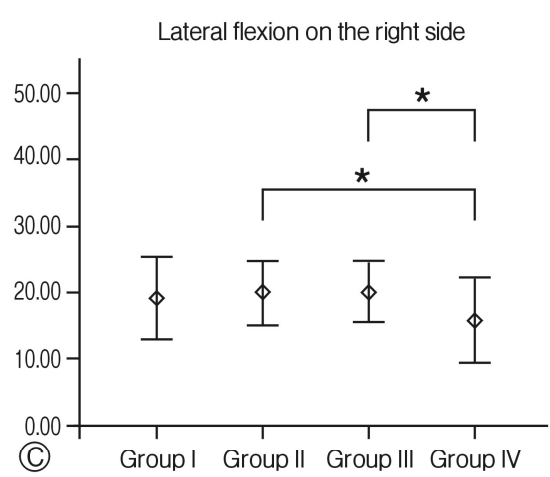

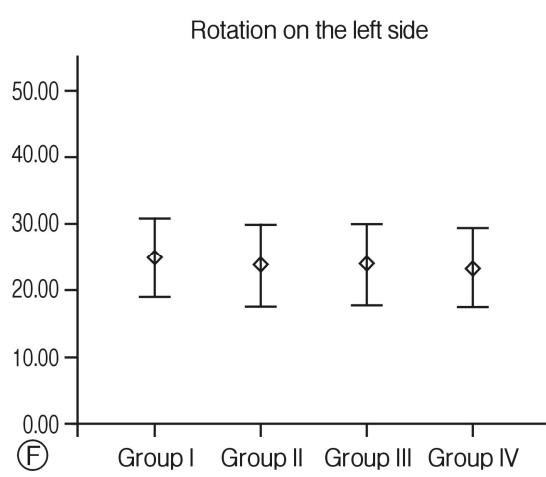

Fig. 1. Results of range of motion in the lumbar spine. Group I: fusion at the L4/5 or L3/4/5 level; group II: fusion at the L5/S1 level; group III: fusion at the L4/5/S1 level; and group IV: fusion at other levels from T7 to L4. 
$\pm 6.47^{\circ}$ in group IV (Fig. 1C, D). The ROM of lumbar lateral flexion had a tendency of being affected by fusion at the $\mathrm{L} 4 / 5$ or L5/S1 level; a statistical significance was observed between groups II and IV in right side bending ( $\mathrm{p}=0.037)$, and between groups III and IV in right side bending $(\mathrm{p}=0.029)$. Respective ROMs of lumbar lateral rotation to the left and right sides were $25.17 \pm 5.86^{\circ}$ and $24.83 \pm 6.25^{\circ}$ in group I, $23.96 \pm 6.25^{\circ}$ and $25.21 \pm 6.16^{\circ}$ in group II, $24.11 \pm 6.09^{\circ}$ and $26.07 \pm 4.78^{\circ}$ in group III, and $23.61 \pm 6.14^{\circ}$ and $22.78 \pm 6.47^{\circ}$ in group IV (Fig. 1E, F). The ROM of lumbar lateral rotation was not affected by fusion at the $\mathrm{L} 4 / 5$ or $\mathrm{L} 5 / \mathrm{S} 1$ level.

\section{DISCUSSION}

The lumbar spine is a major structure responsible for the weight-bearing of the body. At the lumbar spine level, flexionextension movements increased at the caudal levels from $12^{\circ}$ to $14^{\circ}$ in L1-2 and to $17^{\circ}$ in L5-S1, as summarized in Table $2^{18)}$.

Before we conducted this study, it was thought that L5-S1 segment contributes more to lumbar flexion movements than the $\mathrm{L} 45$ segment. Thus, we assumed that the limitations of ROM in the L5-S1 segment group were more restrictive than those in the L4-5 segment group. However, in our study, a significant difference in the ROM of lumbar flexion was not observed between the groups, except for groups I and IV. Especially, no significance in the result between groups I and II suggests that $\mathrm{L} 4 / 5$ or $\mathrm{L} 3 / 4 / 5$ and $\mathrm{L} 5 / \mathrm{S} 1$ segments contribute similarly to flexion movements, which falsifies our assumption.

One study reported that upper lumbar spinal segments contribute to flexion/extension rather than lower lumbar spine segments in the normal spine ${ }^{1)}$. Accordingly, this finding helps

Table 1. Demographic data of 156 patients with thoracolumbar and lumbar fusion

\begin{tabular}{lcl}
\hline \hline & No. of patients (n=156) & \multicolumn{1}{c}{ Fusion level } \\
\hline Group I & $86(55.1 \%)$ & $\mathrm{L} 4 / 5$ or $\mathrm{L} 3 / 4 / 5$ \\
Group II & $24(15.4 \%)$ & $\mathrm{L} 5 / \mathrm{S} 1$ \\
Group III & $34(21.8 \%)$ & $\mathrm{L} 3 / 4 / 5 / \mathrm{S} 1$ or $\mathrm{L} 4 / 5 / \mathrm{S} 1$ \\
Group IV & $12(7.7 \%)$ & $\mathrm{T} 10-\mathrm{S} 1$ \\
\hline
\end{tabular}

explains why there was no difference among groups depending on the lower lumbar segments; however, group IV, which included the most upper lumbar segments, showed no significant difference between the other groups. Therefore, upper lumbar segments do not always contribute to flexion/extension movements. Lateral bending motion did not change differently according to the levels with an average of $7^{\circ}$ to $9^{\circ}$, except the L5-S1 level, as shown in Table $2^{7,18)}$. Lateral bending motion increased with an average of $7^{\circ}$ to $9^{\circ}$, which is not significantly different compared to the other levels of upper lumbar segments. Significant differences among groups IV, II, and III in our study correspond to findings of previous studies that involved upper lumbar segments of group IV. However, the statistical significance observed in only lateral flexion on the right side is unclear, and this may be explained by human errors. Rotational motion was barely observed with an average of $2^{\circ}$ because of the restriction of the horizontal facet angle.

The normal ROM in the lumbar spine is of crucial importance for extensive coupling as well as for the strong relationship between motion and the development of degenerative changes ${ }^{7}$. The most clinically relevant pattern is that of axial rotation and lateral bending, which is important for maintaining the global dynamic balance of the body, and it is relevant in the development of degenerative disc disease ${ }^{14,15)}$. Thus, it is important to improve our understanding and to establish guidelines for diagnosing lumbar pathology ${ }^{16)}$.

Flexion-extension increased caudally from $12^{\circ}$ to $14^{\circ}$ at L1 and to $18^{\circ}$ at L5-S1, under normal circumstances. Lateral bending changed slightly, averaging $7^{\circ}$ to $9^{\circ}$ throughout the lumbar spine. Similarly, rotation was consistent and extremely limited, averaging $2^{\circ}$ per level. In the seldom-studied area of translation, which is extremely important when diagnosing degenerative instability of the lumbar spine, $2 \mathrm{~mm}$ is probably normal for the lumbar spine ${ }^{15)}$.

Physiologic motion of the spine is supported by various paraspinal structures, such as the musculature, disc, facet joint, and ligaments ${ }^{2,12}$. The anterior muscle group flexes the spine, oblique muscles cause spinal rotation, and the psoas muscle group is involved in lateral bending and rotation. Indeed, superficial and deep posterior muscles are involved in extension of the spine; most muscles are also involved in rotation or

Table 2. Different normal values of range of motion

\begin{tabular}{|c|c|c|c|}
\hline & Korean Spinal Neurosurgery $^{\text {(8) }}$ & Pearcy and Tibrewal ${ }^{|5|} /$ Pearcy et al. ${ }^{(4)}$ & Dvorak et al. ${ }^{5}$ \\
\hline L1-L2 & $12^{\circ} / 6^{\circ} / 2^{\circ}$ & $13^{\circ} / 10^{\circ} / 2^{\circ}$ & $11.9^{\circ} / 10.4^{\circ}$ \\
\hline L2-L3 & $14^{\circ} / 6^{\circ} / 2^{\circ}$ & $13^{\circ} / 11^{\circ} / 2^{\circ}$ & $14.5^{\circ} / 12.4^{\circ}$ \\
\hline L3-L4 & $15^{\circ} / 8^{\circ} / 2^{\circ}$ & $13^{\circ} / 10^{\circ} / 2^{\circ}$ & $15.3^{\circ} / 12.4^{\circ}$ \\
\hline L4-L5 & $16^{\circ} / 6^{\circ} / 2^{\circ}$ & $16^{\circ} / 10^{\circ} / 3^{\circ}$ & $18.2^{\circ} / 9.5^{\circ}$ \\
\hline L5-S1 & $17^{\circ} / 3^{\circ} / 1^{\circ}$ & $14^{\circ} / 6^{\circ} / 3^{\circ}$ & $17.0^{\circ} / 5.1^{\circ}$ \\
\hline L4-L5-SI ${ }^{*}$ & $33^{\circ} / 9^{\circ} / 3^{\circ}$ & $30^{\circ} / 16^{\circ}$ & $35.2^{\circ} / 14.6^{\circ}$ \\
\hline
\end{tabular}

The data is presented as flexion-extension/lateral bending/lateral rotation.

*The range of motion was calculated from the sum of L4-5 and L5-S1. 
lateral bending. In the posterior group, superficial muscles are more prominent in bending, and deeper muscles are more prominent in spinal rotation. The muscles found in the lateral to the spinal column are involved in lateral rotation and bending. The effect of the disc on axial rotation would seem prominent, but this has not been assessed experimentally in Korea. The facet appears to provide stability, primarily against anterior translation between the upper thoracic levels. Where the facets change in orientation to the more lumbar pattern, more anterior translation can occur, and axial rotation is more limited. The annulus of the disc, which is the well-developed posterior longitudinal ligament, and the thick ligament flavum prevent hyper-flexion; additionally, the anterior longitudinal ligament resists excessive extension. This is why the posterior elements and ligaments are important in the maintenance of spinal stability. It has been established that posterior spinal surgery damages the paraspinal musculature, facet joint, and ligaments, causing limitations of $\mathrm{ROM}^{9,17)}$. Thus, in clinical practice, postoperative posterior lumbar muscle damage plays an important role in the limitations of ROM.

The normal ROMs are slightly different according to different reports (Table 2). This can affect the disability rating for medical insurance. The recent Korean disability scale categorized the disability rating according to the fusion level after spinal fusion, not according to dynamic spinal motion ${ }^{11)}$. In the clinical setting, the suspected motion limitations are checked differently compared to the real motion limitation. Few biomechanical studies have evaluated pedicle fixation of the lower lumbar spine. One study investigator used an instability model that excluded the posterior ligaments and injury of the disk at the L5-S1 level ${ }^{13)}$. Three-dimensional vertebral motions were measured using a marker on each vertebra: flexion, extension, lateral bending, and axial torque moments. They found that flexion was reduced by $50 \%$ from the intact state, and the stiffness values were higher than those in the intact spine for all the devices. With extension loading, the device restored the stiffness of the vertebrae to at least the normal state. In pure axial loading, the device reduced motion from $75 \%$ to $50 \%$ in the intact state, with statistical significance. This recent study also reported that the limitation of ROM was observed differently with suspected data ${ }^{12,13,18,19)}$. Indeed, each ROM (flexion, extension, bending, and rotation) was affected differently by fusion at the L4/5 and/or L5/S1 level. The modern Korean disability scale might underestimate clinical symptoms so the disability of postoperative patients may be considered a mild limitation of ROM. Therefore, the new rating of disability might indicate a more severe limitation of ROM.

Although we clearly explained the complication of limitations in ROM and discomfort to the patients before spinal surgery, it is not easy for patients to comprehend the limitations of $\mathrm{ROM}$ and how it may affect their activities of daily living or occupation. Therefore, our study may help neurosurgeons explain the many possible outcomes to patients so they understand how the limitations of ROM may affect their daily life.

Although this recent study reviewed a large patient population, there were some limitations. First, this study had no normal control group for comparing the ROM. Instead, the patients with upper thoracolumbar or lumbar fusion were included in group IV, but the results do not precisely represent data from normal individuals. Second, the measurement of ROM was determined by specialists in Rehabilitation. Although this method for measuring the ROM was a sufficient blind test, it impaired standardization because of the possibility of different human errors. Other studies of fleximeter or goniometry showed a poor correlation between ROM measurements, and this limitation should be overcome in a future study with an improved design ${ }^{10)}$. Despite these limitations, this study provided unique and interesting information about the limitation of ROM after lower lumbar fusion operation, which was questioned by many spinal surgeons.

\section{CONCLUSION}

Limitations of ROM always develop after spinal fusion, resulting in discomfort for patients. Thus, it is important to predict future complications depending on spinal fusion levels and to help explain possible limitations of ROM to patients preoperatively.

The results here in suggest that the lower lumbar segments (L4/5 and especially L5/S1) contribute to spinal ROM (extension and lateral flexion), but these segments alone do not play significant roles in spinal flexion movements. Therefore, spinal neurosurgeons should consider the limitations of ROM and that inclusion of the $\mathrm{L} 5 / \mathrm{S} 1$ segment in spinal surgery results in more limitations. Thus, these limitations should be carefully considered in advance.

\section{CONFLICT OF INTEREST}

No potential conflict of interest relevant to this article was reported.

\section{ACKNOWLEDEMENT}

This work was supported by the Inha University Hospital Research Grant.

\section{REFERENCES}

1. Bali T, Kumar MN: Relative contribution of upper and lower lumbar spinal segments to flexion/extension: Comparison between normal spines and spines with disc disease in Asian patients. Asian Spine J 9:770-775, 2015

2. Barr KP, Griggs M, Cadby T: Lumbar stabilization: core con- 
cepts and current literature, Part 1. Am J Phys Med Rehabil 84:473-480, 2005

3. Chun K, Yang I, Kim N, Cho D: Effect of device rigidity and physiological loading on spinal kinematics after dynamic stabilization: An in-vitro biomechanical study. J Korean Neurosurg Soc 58:412-418, 2015

4. Cook DJ, Yeager MS, Cheng BC: Range of motion of the intact lumbar segment: a multivariate study of 42 lumbar spines. Int J Spine Surg 9:5, 2015

5. Dvorak J, Panjabi MM, Chang DG, Theiler R, Grob D: Functional radiographic diagnosis of the lumbar spine. Flexion-extension and lateral bending. Spine (Phila Pa 1976) 16:562-571, 1991

6. Hedman TP, Kostuik JP, Fernie GR, Hellier WG: Design of an intervertebral disc prosthesis. Spine (Phila Pa 1976) 16:S256S260, 1991

7. Izzo R, Guarnieri G, Guglielmi G, Muto M: Biomechanics of the spine. Part II: spinal instability. Eur J Radiol 82:127-138, 2013

8. Kroeze RJ, van der Veen AJ, van Royen BJ, Bank RA, Helder $\mathrm{MN}$, Smit TH: Relation between radiological assessment and biomechanical stability of lumbar interbody fusion in a large animal model. Eur Spine J 22:2731-2739, 2013

9. Malakoutian M, Street J, Wilke HJ, Stavness I, Dvorak M, Fels S, et al.: Role of muscle damage on loading at the level adjacent to a lumbar spine fusion: a biomechanical analysis. Eur Spine J 25:2929-2937, 2016

10. Mannion AF, Klein GN, Dvorak J, Lanz C: Range of global motion of the cervical spine: intraindividual reliability and the influence of measurement device. Eur Spine J 9:379-385, 2000

11. Ministry of Health and Welfare. Criteria for evaluation of degree of disability. Sejong: Ministry of Health and Welfare, 2017
(http://www.mohw.go.kr/react/gm/sgm0601vw.jsp?PAR_MEN U_ID $=13 \& M E N U$ ID $=1304020601 \& C O N T \_S E Q=293271$ \&page =5) [Accessed May 1, 2018]

12. Panjabi MM: The stabilizing system of the spine. Part I. Function, dysfunction, adaptation, and enhancement. J Spinal Disord 5:383-389, 1992

13. Panjabi MM, Yamamoto I, Oxland TR, Crisco JJ, Freedman D: Biomechanical stability of five pedicle screw fixation systems in a human lumbar spine instability model. Clin Biomech (Bristol, Avon) 6:197-205, 1991

14. Pearcy M, Portek I, Shepherd J: Three-dimensional X-ray analysis of normal movement in the lumbar spine. Spine (Phila Pa 1976) 9:294-297, 1984

15. Pearcy MJ, Tibrewal SB: Axial rotation and lateral bending in the normal lumbar spine measured by three-dimensional radiography. Spine (Phila Pa 1976) 9:582-587, 1984

16. Shin JH, Wang S, Yao Q, Wood KB, Li G: Investigation of coupled bending of the lumbar spine during dynamic axial rotation of the body. Eur Spine J 22:2671-2677, 2013

17. Strube P, Putzier M, Streitparth F, Hoff EK, Hartwig T: Postoperative posterior lumbar muscle changes and their relationship to segmental motion preservation or restriction: a randomized prospective study. J Neurosurg Spine 24:25-31, 2016

18. The Korean Spinal Neurosurgery Society: The textbook of spine, ed 2nd. Paju: Koonja, 2013

19. White AA, Panjabi MM: Clinical biomechanics of the spine, ed 2nd. Philadelphia, PA: Lippincott, 1990

20. Yamamoto I, Panjabi MM, Crisco T, Oxland T: Three-dimensional movements of the whole lumbar spine and lumbosacral joint. Spine (Phila Pa 1976) 14:1256-1260, 1989 